\title{
Differential flatness theory and Extended Kalman Filtering for sensorless control of induction motors
}

\author{
Gerasimos Rigatos \\ Department of Engineering \\ Harper Adams University College \\ TF10 8NB, Shropshire, UK \\ Email: grigat@ieee.org
}

\author{
Pierluigi Siano \\ Department of Industrial Engineering \\ University of Salerno \\ 84084, Fisciano, Italy \\ Email: psiano@unisa.it
}

\begin{abstract}
Diffenential flatness theory and Extended Kalman Filtering (EKF) are used for implementing a sensorless control scheme for induction motor drives. It is shown that the induction motor is a differentially flat system, since all state variables of the circuits describing the motor's dynamics can be expressed as functions of the flat output and its derivatives. The flat output consists of the angle of the rotor and of the the angle of the magnetic field. Flatness-based control for the complete (sixthorder) induction motor model is analyzed while the Extended Kalman Filter is proposed to estimate the state vector of the nonlinear electric motor using a limited number of sensors. The efficiency of the above mentioned Extended Kalman Filter-based control scheme, is tested through simulation experiments.
\end{abstract}

\section{INTRODUCTION}

During the last years, there has been significant effort in improving the performance of induction motors. The applications of induction motors (IM) are mainly concerned with motion transmission and transportation systems. Induction motors have been the most widely used machines in fixed-speed applications for reasons of cost, size, weight, reliability, ruggedness, simplicity, efficiency, and ease of manufacture. With the field-oriented method, the dynamic behavior of the induction motor is rather similar to that of a separately excited DC motor [1]. The possibility to reduce the number of sensors involved in the control of electric motors has been a subject of systematic research during the last years [2-6]. As a result, state estimation-based control has become an active research area in the field of electric machines and power electronics. To reconstruct the state vector of the induction motor, from a sequence of angular position measurements and the associated voltage or current input measurements, state estimation/filtering has to be used.

The Kalman Filter provides state estimates of improved accuracy despite noise (stochastic disturbances) affecting the system's dynamics, if the noise covariance is known [7-8]. Actually, for linear systems subject to Gaussian measurement or process noise the Kalman Filter is the optimal state estimator, since it results in minimization of the trace of the estimation error's covariance matrix [9-11]. For nonlinear systems, subject to Gaussian noise one can use the generalization of the Kalman Filter as formulated in terms of the Extended Kalman Filter (EKF). The Extended Kalman
Filter is based on a linearization of the systems' dynamics, round the current state estimate, using a first order Taylor expansion [12].

The current paper studies sensorless control for induction motors using differential flatness-theory and Extended Kalman Filtering techniques. The structure of the paper is as follows: In Section II, the induction motor model and the associated reference frames are analyzed. In Section III, flatness-based control for the complete (sixth-order) induction motor model is developed. Unlike field-oriented control no assumption is made about decoupling between the motor's speed and the flux dynamics. In Section IV the EKF is proposed to estimate the state vector of the nonlinear electric motor using a limited number of sensors, and control of the induction motor is implemented through feedback of the estimated state vector. In Section $\mathrm{V}$ the efficiency of the above mentioned EKF-based control scheme, for induction motor models, is tested through simulation experiments. Finally, in Section VI concluding remarks are given.

\section{CONTROL OF THE FIELD-ORIENTED INDUCTION MOTOR}

\section{A. Field-oriented induction motor model}

To derive the dynamic model of an induction motor the threephase variables are first transformed to two-phase ones. This two-phase system can be described in the stator-coordinates frame $\alpha-b$, and the associated voltages are denoted as $v_{s_{\alpha}}$ and $v_{s_{b}}$, while the currents of the stator are $i_{s_{\alpha}}$ and $i_{s_{b}}$, and the components of the rotor's magnetic flux are $\psi_{r_{\alpha}}$ and $\psi_{r_{b}}$. Then, the rotation angle of the rotor with respect to the stator is denoted by $\delta$. Next, the rotating reference frame $d-q$ on rotor, is defined (Fig. 1).

The state vector of the motor is defined as $x=$ $\left[\theta, \omega, \psi_{r_{\alpha}}, \psi_{r_{b}}, i_{s_{\alpha}}, i_{s_{b}}\right]$ and the dynamic model of the induction motor is written as [5]:

$$
\begin{gathered}
\dot{x}=f(x)+g_{\alpha}(x) v_{s_{\alpha}}+g_{b}(x) v_{s_{b}}+w(t) \\
z=h(x)+v(t)
\end{gathered}
$$

with the first row to describe the state equation of the motor and the second row to described the measurement equation of the motor. The process noise $w(k)$ given in Eq. (1) is due 


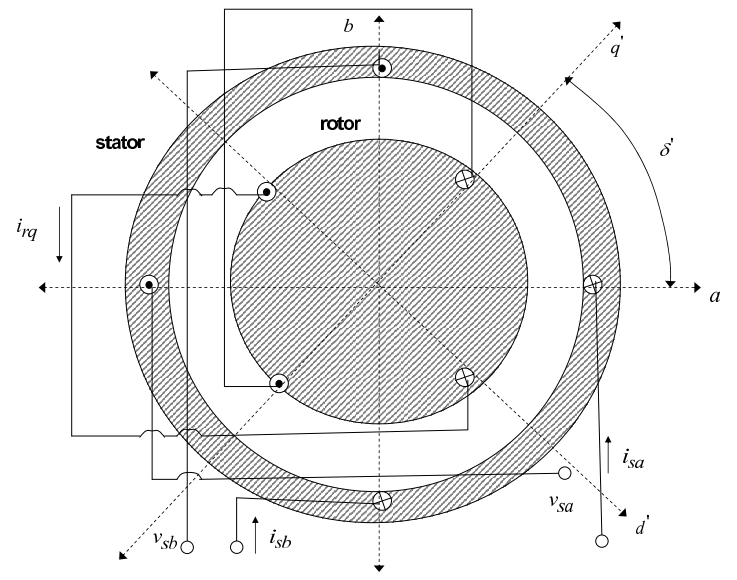

Fig. 1. AC motor circuit, with the $a-b$ stator reference frame and the $d-q$ rotor reference frame

to model inaccuracies associated with random variations of the model's parameters. For example resistances, inductances and magnetic permeability of the electric motor can exhibit a stochastic variation round a nominal value. The measurement noise $v(k)$ given in Eq. (1) is due to stochastic variations of the elements of the measuring devices. It is noted that if the effects of the noise signals are not compensated by a filtering procedure, the performance of the control loop can be unsatisfactory or even the stability of the control loop can be risked.

In the case of the induction motor model the measured variables are the rotor's angle and the $a-b$ reference frame currents of the stator.

$$
\begin{gathered}
f(x)=\left(\begin{array}{c}
x_{2} \\
\mu_{1}\left(x_{3} x_{6}-x_{4} x_{5}\right)-\frac{T_{L}}{J} \\
-\alpha_{1} x_{3}-n_{p} x_{2} x_{4}+\alpha_{1} M x_{5} \\
n_{p} x_{2} x_{3}-\alpha_{1} x_{4}+\alpha_{1} M x_{6} \\
\alpha_{1} \beta_{1} x_{3}+n_{p} \beta_{1} x_{2} x_{4}-\gamma x_{5} \\
-n_{p} \beta_{1} x_{2} x_{3}+\alpha_{1} \beta_{1} x_{4}-\gamma_{1} x_{6}
\end{array}\right) \\
g_{\alpha}=\left[0,0,0,0, \frac{1}{\sigma L_{s}}, 0\right]^{T} \quad g_{b}=\left[0,0,0,0,0, \frac{1}{\sigma L_{s}}\right]^{T}
\end{gathered}
$$

$J$ is the rotor's inertia, and $T_{L}$ is the external load torque. The rest of the model parameters are $\sigma=1-M^{2} / L_{s} L_{r}$, $\alpha_{1}=\frac{R_{r}}{L_{r}}, \beta_{1}=\frac{M}{\sigma L_{s} L_{r}}, \gamma_{1}=\left(\frac{M^{2} R_{r}}{\sigma L_{s} L_{r}^{2}}+\frac{R_{s}}{\sigma L_{s}}\right), \mu_{1}=\frac{n_{p} M}{J L_{r}}$, where $L_{s}, L_{r}$ are the stator and rotor autoinductances, $M$ is the mutual inductance and $n_{p}$ is the number of poles.

\section{B. Decoupling of speed-flux dynamics}

The classical method for induction motors control is based on a transformation of the stator's currents $\left(i_{s_{\alpha}}\right.$ and $\left.i_{s_{b}}\right)$ and of the magnetic fluxes of the rotor $\left(\psi_{r_{\alpha}}\right.$ and $\left.\psi_{r_{b}}\right)$ to the reference frame $d-q$ which rotates together with the rotor (Fig. 1). In the $d-q$ frame there will be only one non-zero component of the magnetic flux $\psi_{r_{d}}$, while the component of the flux along the $q$ axis equals 0 . The new control inputs of the system are considered to be $v_{s_{d}}, v_{s_{q}}$, and are associated to the $d-q$ frame voltages $v_{d}$ and $v_{q}$, respectively. The control inputs $v_{s_{d}}, v_{s_{q}}$ are connected to $v_{s_{\alpha}}, v_{s_{b}}$ of Eq. (1), according to the relation

$$
\left(\begin{array}{l}
v_{s \alpha} \\
v_{s b}
\end{array}\right)=\|\psi\| \cdot\left(\begin{array}{cc}
\psi_{r_{\alpha}} & \psi_{r_{b}} \\
\psi_{r_{b}} & \psi_{r_{\alpha}}
\end{array}\right)^{-1}\left(\begin{array}{l}
v_{s d} \\
v_{s q}
\end{array}\right)
$$

where $\psi=\psi_{r_{d}}$ and $\|\psi\|=\sqrt{\psi_{s_{\alpha}}^{2}+\psi_{s_{b}}^{2}}$. Next, the following nonlinear feedback control law is defined

$$
\left(\begin{array}{c}
v_{s d} \\
v_{s q}
\end{array}\right)=\sigma L_{s}\left(\begin{array}{c}
-n_{p} \omega i_{s q}-\frac{\alpha M i_{s_{q}}{ }^{2}}{\psi_{r_{d}}}-\alpha b \psi_{r_{d}}+v_{d} \\
n_{p} \omega i_{s d}+b n_{p} \omega \psi_{r_{d}}+\frac{\alpha M i_{s_{q} i_{s_{d}}}}{\psi_{r_{d}}}+v_{q}
\end{array}\right)
$$

The control signal in the coordinates system $\alpha-b$ is

$$
\begin{gathered}
\left(\begin{array}{c}
v_{s \alpha} \\
v_{s b}
\end{array}\right)=\|\psi\| \sigma L_{s}\left(\begin{array}{cc}
\psi_{r_{\alpha}} & \psi_{r_{b}} \\
-\psi_{r b} & \psi_{r_{\alpha}}
\end{array}\right)^{-1} . \\
\cdot\left(\begin{array}{c}
-n_{p} \omega i_{s q}-\frac{\alpha M i_{s_{q}}}{\psi_{r_{d}}}-\alpha \beta \psi_{r_{d}}+v_{d} \\
n_{p} \omega i_{s d}+\beta n_{p} \omega \psi_{r_{d}}+\frac{\alpha M i_{s_{q}} i_{s d}}{\psi_{r_{d}}}+v_{q}
\end{array}\right)
\end{gathered}
$$

Substituting Eq. (6) into Eq. (1) one obtains

$$
\begin{gathered}
\frac{d}{d t} \omega=\mu \psi_{r_{d}} i_{s q}-\frac{T_{L}}{J} \\
\frac{d}{d t} i_{s q}=-\gamma i_{s q}+v_{q} \\
\frac{d}{d t} \psi_{r_{d}}=-\alpha \psi_{r_{d}}+\alpha M i_{s d} \\
\frac{d}{d t} i_{s d}=-\gamma i_{s d}+v_{d} \\
\frac{d}{d t} \rho=n_{p} \omega+\alpha M \frac{i_{s q}}{\psi_{r d}}
\end{gathered}
$$

The system of Eq. (7) to Eq. (11) consists of two linear subsystems, where the first one has as output the magnetic flux $\psi_{r_{d}}$ and the second has as output the rotation speed $\omega$, i.e.

$$
\begin{gathered}
\frac{d}{d t} \psi_{r_{d}}=-\alpha \psi_{r_{d}}+\alpha M i_{s d} \\
\frac{d}{d t} i_{s d}=-\gamma i_{s d}+v_{d} \\
\frac{d}{d t} \omega=\mu \psi_{r_{d}} i_{s q}-\frac{T_{L}}{J} \\
\frac{d}{d t} i_{s q}=-\gamma i_{s q}+v_{q}
\end{gathered}
$$

If $\psi_{r_{d}} \rightarrow \psi_{r_{d}}^{\text {ref }}$, i.e. the transient phenomena for $\psi_{r_{d}}$ have been eliminated and $\psi_{r_{d}}$ has converged to a steady state value, then Eq. (14) is not dependent on $\psi_{r_{d}}$, and consequently the two subsystems described by Eq. (12)-(13) and Eq. (14)-(15) are decoupled.The subsystem that is described by Eq. (12) and Eq. 
(13) is linear and has as control input $v_{d}$, and can be controlled using methods of linear control. For instance the following PI controller has been proposed for the control of the magnetic flux [13]

$$
v_{d}(t)=-k_{d 1}\left(\psi_{r_{d}}-\psi_{r_{d}}^{\mathrm{ref}}\right)-k_{d 2} \int_{0}^{t}\left(\psi_{r_{d}}(\tau)-\psi_{r_{d}}{ }^{\mathrm{ref}}(\tau)\right) d \tau
$$

If the control of Eq. (16) is applied to the subsystem that is described by Eq. (12) and Eq. (13), then one can succeed $\psi_{r_{d}}(t) \rightarrow \psi_{r_{d}}^{\text {ref }}(t)$. If $\psi_{r_{d}}(t)$ cannot be measured sufficiently using Hall sensors then it can be reconstructed using some kind of observer.

Next, the subsystem that consists of Eq. (14) and Eq. (15) can be controlled. The term $T=\mu \psi_{r_{d}}{ }^{\text {ref }} i_{s q}$ denotes the torque developed by the motor. The above mentioned subsystem is a model equivalent to that of a DC motor and thus after succeeding $\psi_{r_{d}} \rightarrow \psi_{r_{d}}^{\text {ref }}$, one can also control the motor's speed $\omega$, using control algorithms already applied to the control of DC motors [1].

\section{A FLATNESS-BASED CONTROL APPROACH FOR INDUCTION MOTORS}

In [14] the voltage-fed induction machine was shown to be a differentially flat system. It has been shown that the angle of the rotor position (rotation angle $\theta$ ) and the angle $\rho$ of the magnetic field (angle between flux $\psi_{a}$ and $\psi_{b}$ ) constitute a flat output for the induction motor model [14],[15-17]. Since all state variables of the circuits describing the induction motor dynamics can be expressed as functions of $y=(\theta, \rho)$ and its derivatives it can be concluded that the induction motor is a differentially flat system.

The equations of the induction motor in the $d-q$ reference frame, given by Eq. (12) to Eq. (15), are now rewritten in the form of Eq. (17) to Eq. (21):

$$
\begin{gathered}
\frac{d}{d t} \omega=\mu \psi_{r_{d}} i_{s q}-\frac{T_{L}}{J} \\
\frac{d}{d t} \psi_{r_{d}}=-\alpha \psi_{r_{d}}+\alpha M i_{s d} \\
\frac{d}{d t} i_{s d}=-\gamma i_{s d}+\alpha \beta \psi_{r_{d}}+n_{p} \omega i_{s q}+\frac{\alpha M i_{s q}{ }^{2}}{\psi_{r_{d}}}+\frac{1}{\sigma L_{s}} v_{s d} \\
\frac{d}{d t} i_{s q}=-\gamma i_{s q}-\beta n_{p} \omega \psi_{r_{d}}-n_{p} \omega i_{s d}-\frac{\alpha M i_{s q} i_{s d}}{\psi_{d}}+\frac{1}{\sigma L_{s}} v_{s q} \\
\frac{d}{d t} \rho=n_{p} \omega+\frac{\alpha M i_{s q}}{\psi_{r_{d}}}
\end{gathered}
$$

The flat outputs for the voltage-fed induction motor are the angle of the rotor $\theta$ and variable $\rho$, where $\rho$ has been defined as the rotor flux angle. According to [17], if the stator current dynamics are much faster than the speed and flux dynamics a faster inner current control loop can be designed using only Eq. (19) and Eq. (20) and assuming the speed and flux as constants. For the outer speed and flux control design the stator currents are treated as new control inputs and the system behavior is described by Eq. (17), Eq. (18) and Eq. (21). This system of lower order is also flat with $\psi_{r_{d}}$ and $\theta$ as flat outputs. It can be shown that all state variables of the induction motor can be written as functions of the flat outputs and their derivatives. Moreover, using Eq. (19) and Eq. (20) a controller that satisfies the flatness properties (and thus it can be also expressed as a function of the flat outputs and their derivatives) is defined as follows:

$$
\begin{aligned}
& v_{s_{d}}=\sigma L_{s}\left(\frac{d i_{s_{d}}^{*}}{d t}+\gamma i_{s_{d}}^{*}-\alpha \beta \psi_{r_{d}}-n_{p} \omega i_{s_{q}}-\frac{\alpha M{i_{s_{q}}}^{2}}{\psi_{r_{d}}}+v_{d}\right) \\
& v_{s_{q}}=\sigma L_{s}\left(\frac{d i_{s_{q}}^{*}}{d t}+\gamma i_{s_{q}}^{*}+\beta n_{p} \omega \psi_{r_{d}}+n_{p} \omega i_{s_{d}}+\frac{\alpha M i_{s_{q}} i_{s_{d}}}{\psi_{r_{d}}}+v_{q}\right)
\end{aligned}
$$

where $i_{s_{q}}^{*}$ and $i_{s_{d}}^{*}$ denote current setpoints. Substituting Eq. (22) and Eq. (23) into Eq. (19) and Eq. (20) one obtains the dynamics of the current tracking errors.

$$
\begin{aligned}
& \frac{d \Delta i_{s_{d}}}{d t}=-\gamma \Delta i_{s_{d}}+v_{d} \\
& \frac{d \Delta i_{s_{q}}}{d t}=-\gamma \Delta i_{s_{q}}+v_{q}
\end{aligned}
$$

where $\Delta i_{s_{d}}=\left(i_{s_{d}}-i_{s_{d}}^{*}\right)$. For the decoupled system of Eq. (24) and Eq. (25) one can apply state feedback control. For example a suitable state feedback controller would be

$$
\begin{gathered}
v_{d}=-\gamma_{1} \Delta i_{s_{d}} \\
v_{q}=-\gamma_{2} \Delta i_{s_{q}}
\end{gathered}
$$

Tracking of the reference setpoint can be also succeeded for the rotor's speed and flux through the application of the control law of Eq. (22) and Eq. (23) to Eq. (17) and Eq. (21). The control inputs are chosen as

$$
\begin{gathered}
i_{s_{d}}=\frac{1}{\alpha M}\left(\frac{d \psi_{r_{d}}{ }^{*}}{d t}+\alpha \psi_{r_{d}}^{*}+i_{d}\right) \\
i_{s_{q}}=\frac{1}{\mu \psi_{r_{d}}}\left(\frac{d \omega^{*}}{d t}+i_{q}\right)
\end{gathered}
$$

Denoting $\Delta \psi_{r_{d}}=\psi_{r_{d}}-\psi_{r_{d}}^{*}$ and $\Delta \omega=\omega-\omega^{*}$ the tracking error dynamics are given by

$$
\begin{gathered}
\frac{d \Delta \psi_{r_{d}}}{d t}=-\alpha \Delta \psi_{r_{d}}+i_{d} \\
\frac{d \Delta \omega}{d t}=-\frac{T}{J}+i_{q}
\end{gathered}
$$

The convergence of the tracking error to zero can be assured through the application of the following feedback control laws: 


$$
\begin{gathered}
i_{d}=-\alpha_{1} \Delta \psi_{r_{d}} \\
i_{q}=\frac{T}{J}-\alpha_{2} \Delta \omega
\end{gathered}
$$

\section{EXTENDED KALMAN FILTER FOR THE NONLINEAR INDUCTION MOTOR MODEL}

The following nonlinear time-invariant state model is now considered [11]:

$$
\begin{gathered}
x(k+1)=\phi(x(k))+L(k) u(k)+w(k) \\
z(k)=\gamma(x(k))+v(k)
\end{gathered}
$$

where where $x \in R^{m \times 1}$ is the system's state vector, and $z \in R^{p \times 1}$ is the system's output, while $w(k)$ and $v(k)$ are uncorrelated, Gaussian zero-mean noise processes with covariance matrices $Q(k)$ and $R(k)$ respectively. The operators $\phi(x)$ and $\gamma(x)$ are $\phi(x)=\left[\phi_{1}(x), \phi_{2}(x), \cdots, \phi_{m}(x)\right]^{T}$, and $\gamma(x)=\left[\gamma_{1}(x), \gamma_{2}(x), \cdots, \gamma_{p}(x)\right]^{T}$, respectively. It is assumed that $\phi$ and $\gamma$ are sufficiently smooth in $x$ so that each one has a valid series Taylor expansion. Following a linearization procedure, $\phi$ is expanded into Taylor series about $\hat{x}$, which gives $\phi(x(k))=\phi(\hat{x}(k))+J_{\phi}(\hat{x}(k))[x(k)-\hat{x}(k)]+\cdots$, where $J_{\phi}(x)$ is the $m \times m$ Jacobian of $\phi$ calculated at $\hat{x}(k)$ :

$$
J_{\phi}(x)=\left.\frac{\partial \phi}{\partial x}\right|_{x=\hat{x}(k)}=\left(\begin{array}{cccc}
\frac{\partial \phi_{1}}{\partial x_{1}} & \frac{\partial \phi_{1}}{\partial x_{2}} & \cdots & \frac{\partial \phi_{1}}{\partial x_{m}} \\
\frac{\partial \phi_{2}}{\partial x_{1}} & \frac{\partial \phi_{2}}{\partial x_{2}} & \cdots & \frac{\partial \phi_{2}}{\partial x_{m}} \\
\vdots & \vdots & \vdots & \vdots \\
\frac{\partial \phi_{m}}{\partial x_{1}} & \frac{\partial \phi_{m}}{\partial x_{2}} & \cdots & \frac{\partial \phi_{m}}{\partial x_{m}}
\end{array}\right)
$$

Likewise, $\gamma$ is expanded about $\hat{x}^{-}(k)$, i.e. $\gamma(x(k))=$ $\gamma\left(\hat{x}^{-}(k)\right)+J_{\gamma}\left[x(k)-\hat{x}^{-}(k)\right]+\cdots$, where $\hat{x}^{-}(k)$ is the estimation of the state vector $x(k)$ before measurement at the $k$-th instant to be received and $\hat{x}(k)$ is the updated estimation of the state vector after measurement at the $k$-th instant has been received. The $p \times m$ Jacobian $J_{\gamma}(x)$ is

$$
J_{\gamma}(x)=\left.\frac{\partial \gamma}{\partial x}\right|_{x=\hat{x}^{-}(k)}=\left(\begin{array}{cccc}
\frac{\partial \gamma_{1}}{\partial x_{1}} & \frac{\partial \gamma_{1}}{\partial x_{2}} & \cdots & \frac{\partial \gamma_{1}}{\partial x_{m}} \\
\frac{\partial \gamma_{2}}{\partial x_{1}} & \frac{\partial \gamma_{2}}{\partial x_{2}} & \cdots & \frac{\partial \gamma_{2}}{\partial x_{m}} \\
\vdots & \vdots & \vdots & \vdots \\
\frac{\partial \gamma_{p}}{\partial x_{1}} & \frac{\partial \gamma_{p}}{\partial x_{2}} & \cdots & \frac{\partial \gamma_{p}}{\partial x_{m}}
\end{array}\right)
$$

The resulting expressions create first order approximations of $\phi$ and $\gamma$. Thus the linearized version of the plant is obtained, i.e. $x(k+1)=\phi(\hat{x}(k))+J_{\phi}(\hat{x}(k))[x(k)-\hat{x}(k)]+w(k)$ and $z(k)=\gamma\left(\hat{x}^{-}(k)\right)+J_{\gamma}\left(\hat{x}^{-}(k)\right)\left[x(k)-\hat{x}^{-}(k)\right]+v(k)$. Now, the Extended Kalman Filter (EKF) recursion is as follows: First, the time update is considered: by $\hat{x}(k)$ the estimation of the state vector at instant $k$ is denoted. Given initial conditions $\hat{x}^{-}(0)$ and $P^{-}(0)$ the recursion proceeds as:

- Measurement update. Acquire $z(k)$ and compute:

$$
\begin{aligned}
& K(k)=P^{-}(k) J_{\gamma}^{T}\left(\hat{x}^{-}(k)\right) \cdot \\
& \cdot\left[J_{\gamma}\left(\hat{x}^{-}(k)\right) P^{-}(k) J_{\gamma}^{T}\left(\hat{x}^{-}(k)\right)+R(k)\right]^{-1} \\
& \hat{x}(k)=\hat{x}^{-}(k)+K(k)\left[z(k)-\gamma\left(\hat{x}^{-}(k)\right)\right] \\
& P(k)=P^{-}(k)-K(k) J_{\gamma}\left(\hat{x}^{-}(k)\right) P^{-}(k)
\end{aligned}
$$

- Time update. Compute:

$$
\begin{aligned}
& P^{-}(k+1)=J_{\phi}(\hat{x}(k)) P(k) J_{\phi}^{T}(\hat{x}(k))+Q(k) \\
& \hat{x}^{-}(k+1)=\phi(\hat{x}(k))+L(k) u(k)
\end{aligned}
$$

The Extended Kalman Filter is applicable to the induction motor described in Sections II and III.

\section{Simulation tests}

The approach on flatness-based control of the induction motor that was presented in Section III requires knowledge of the electric motor's state vector $x=\left[\theta, \omega, \psi_{s_{d}}, i_{s_{d}}, i_{s_{q}}, \rho\right]$. It will be shown that it is possible to implement state estimation for the electric motor using measurements only of the rotation angle $\theta$ and of the stator currents $i_{s_{a}}$ and $i_{s_{b}}$. The Extended Kalman Filter can give estimates of the non-measured state vector elements, i.e. of the rotation speed $\omega$, of the magnetic flux $\psi_{r_{d}}$ and of the angle $\rho$ between the flux vectors $\psi_{r_{a}}$ and $\psi_{r_{b}}$. Measuring currents $i_{s_{a}}$ and $i_{s_{b}}$ and using the estimate of angle $\rho$, the input measurements $i_{s_{d}}$ and $i_{s_{q}}$ can be provided to the Extended Kalman Filter. Thus one has

$$
\left(\begin{array}{l}
i_{s_{d}} \\
i_{s_{q}}
\end{array}\right)=\left(\begin{array}{cc}
\cos (\hat{\rho}) & \sin (\hat{\rho}) \\
-\sin (\hat{\rho}) & \cos (\hat{\rho})
\end{array}\right) \cdot\left(\begin{array}{c}
i_{s_{a}} \\
i_{s_{b}}
\end{array}\right)
$$

The proposed flatness-based control scheme with the use of Extended Kalman Filtering for estimation of the nonmeasurable parameters of the motor's state vector is depicted in Fig. 2.

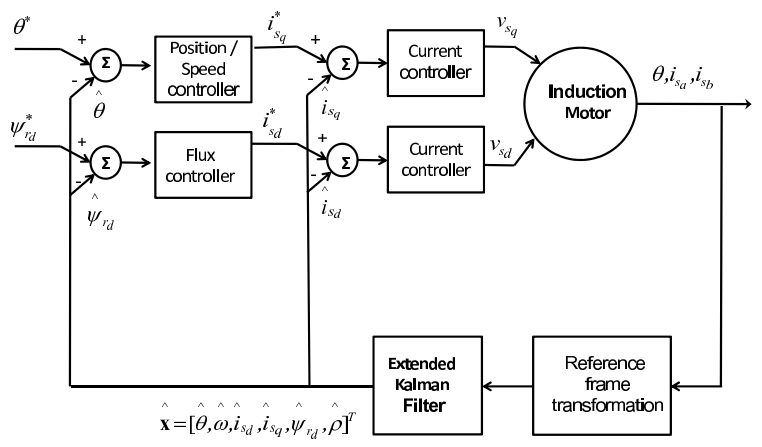

Fig. 2. Schematic diagram the proposed flatness-based control scheme with the use of Extended Kalman Filtering

To implement the Extended Kalman Filter in the induction motor's model that is expressed in the $d-q$ reference frame the Jacobian matrices $J_{\phi}$ and $J_{\gamma}$ are calculated. Thus: 


$$
J_{\phi}=\left[J_{\phi}^{1}, J_{\phi}^{2}, J_{\phi}^{3}, J_{\phi}^{4}, J_{\phi}^{5}, J_{\phi}^{6},\right]^{T}
$$

where the rows of the above defined Jacobian matrix are given by $J_{\phi}^{1}=[0,1,0,0,0,0]$, $J_{\phi}^{2}=\left[0,0, \mu x_{5}, 0, \mu x_{3}, 0\right], \quad J_{\phi}^{3}=[0,0,-\alpha, \alpha M, 0,0]$, $J_{\phi}^{4}=\left[0, n_{p} x_{5}, \alpha \beta-\frac{\alpha M x_{5}^{2}}{x_{3}^{2}},-\gamma, n_{p} x_{2}+\frac{2 \alpha M x_{5}}{x_{3}}, 0\right]$, $J_{\phi}^{5}=\left[0,-\beta n_{p} x_{3}-n_{p} x_{4},-\beta n_{p} x_{2}+\frac{\alpha M x_{4} x_{5}}{x_{3}^{2}},-n_{p} x_{2}-\right.$ $\left.\frac{\alpha M x_{5}}{x_{3}},-\gamma-\frac{\alpha M x_{4}}{x_{3}}, 0\right]$ and $J_{\phi}^{6}=\left[0, n_{p},-\frac{\alpha M x_{5}}{x_{3}^{2}}, 0, \frac{\alpha M}{x_{3}}, 0\right]$.

Moreover, one has

$$
J_{\gamma}=\left(\begin{array}{cccccc}
1 & 0 & 0 & 0 & 0 & 0 \\
0 & 0 & 0 & 1 & 0 & 0 \\
0 & 0 & 0 & 0 & 1 & 0
\end{array}\right)
$$

It is noted that: (i) the discrete-time model of Eq. (34) can be computed from Eq. (1) after applying common discretization methods (e.g. first order Euler approximation of the continuous-time derivative), (ii) if a Jacobian matrix $J_{\phi}$, associated to the drift term of the system's dynamics, is computed using the system's continuous-time description of Eq. (1), then in the EKF recursion of Eq. (37) and (38) it should be substituted by $I+T_{s} J_{\phi}$ where $T_{s}$ is the sampling period and $I \in R^{n \times n}$ is the identity matrix.

Simulation results on flatness-based control of the induction motor when using the EKF for estimation of its state vector from output measurements, and considering increased measurement noise, are presented in Fig. 3 to Fig. 8. The real state variable is denoted by the dashed blue line, the estimated state variable is denoted by the dashed green line, while the associated reference setpoint is denoted by the continuous red line. It can be observed that, although using a reduced number of sensors, the proposed state estimation-based control scheme provides accurate tracking of the reference setpoints.

Taking into account that several variables of the induction motor state vector (e.g. rotation speed and magnetic flux) are not directly measurable (due to sensors cost and limited reliability, sensors failures and difficulties in sensors installation) the significance of state estimation through Kalman Filtering becomes clear. It can be noticed that the Extended Kalman Filter is an efficient approach for the implementation of state estimation-based control of the sixth-order induction motor model. The Unscented Kalman Filter can be also used in place of the Extended Kalman Filter and in the latter case there will be no need to compute Jacobian matrices.

Finally, it is noted that to compensate for external disturbances and parameter variations in state estimation-based nonlinear control schemes the following approaches are possible (i) use of adaptive Kalman Filter, (ii) redesign of the Kalman filter in the form of a disturbance observer, (iii) redesign of the Kalman Filter in the form of a high-gain Extended Kalman Filter [18-20].

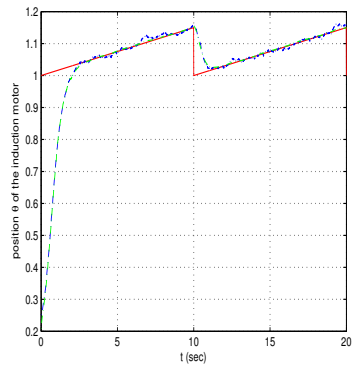

(a)

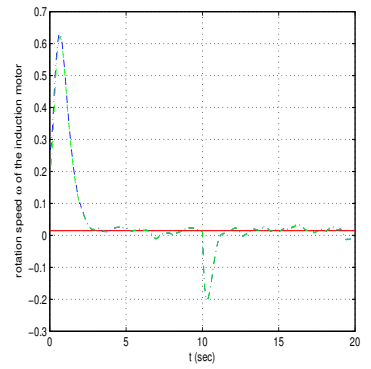

(b)
Fig. 3. Flatness-based control of the induction motor with the use of EKF when tracking a seesaw setpoint (a) rotor's angle $\theta$ (b) rotor's speed $\omega$

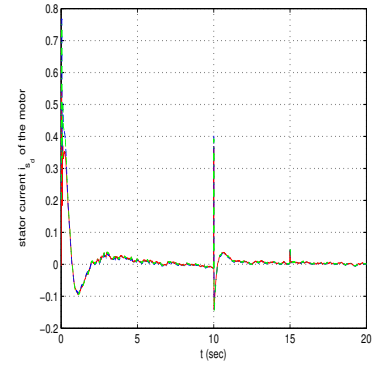

(a)

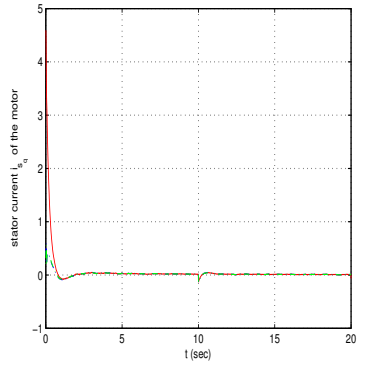

(b)
Fig. 4. Flatness-based control of the induction motor with the use of EKF when tracking a seesaw setpoint (a) stator's current $i_{s_{d}}$ (b) stator's current $i_{s_{q}}$

\section{Conclusions}

The paper has studied sensorless control, for induction motors, using differential flatness theory and Extended Kalman Filtering techniques. It has been shown that the induction motor is a differentially flat system, since all state variables of the circuits describing the motor's dynamics can be expressed as functions of the flat output and its derivatives. The flat output consists of the angle of the rotor and of the angle

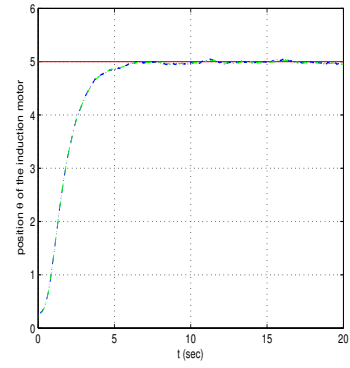

(a)

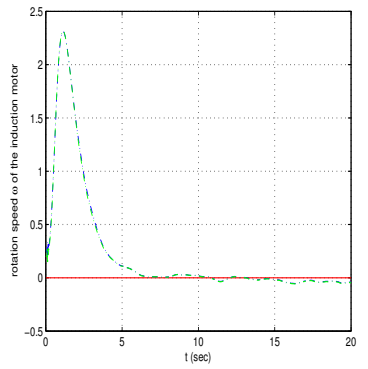

(b)
Fig. 5. Flatness-based control of the induction motor with the use of EKF when tracking a constant setpoint (a) rotor's angle $\theta$ (b) rotor's speed $\omega$ 


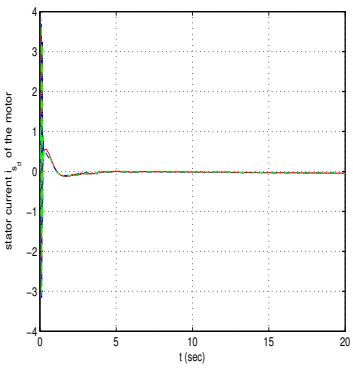

(a)

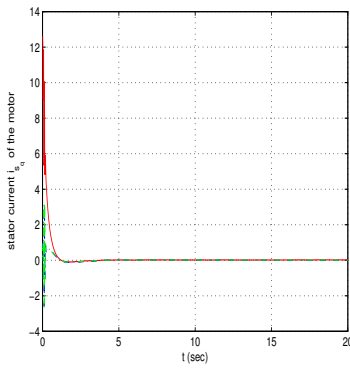

(b)
Fig. 6. Flatness-based control of the induction motor with the use of EKF when tracking a constant setpoint (a) stator's current $i_{s_{d}}$ (b) stator's current $i_{s_{q}}$

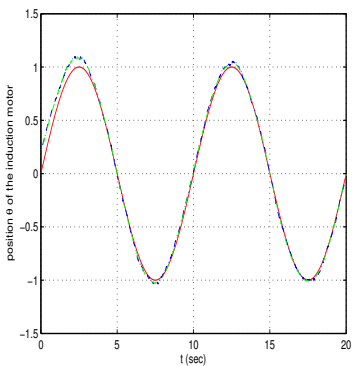

(a)

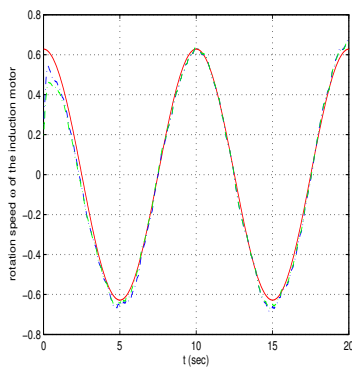

(b)
Fig. 7. Flatness-based control of the induction motor with the use of EKF when tracking a sinusoidal setpoint (a) rotor's angle $\theta$ (b) rotor's speed $\omega$

of the magnetic field. State estimation-based control was developed for the complete (sixth-order) dynamical model of the induction motor using a nonlinear flatness-based controller and the state estimation provided by the Extended Kalman Filter. Flatness-based control for induction motors considers a nested control loops scheme. Taking into account that several variables of the induction motor state vector (e.g. rotation speed and magnetic flux) are not directly measurable (due

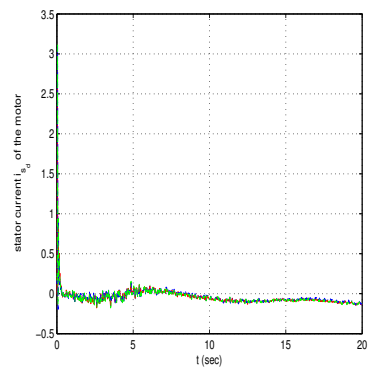

(a)

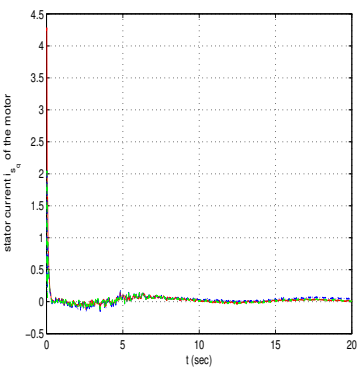

(b)
Fig. 8. Flatness-based control of the induction motor with the use of EKF when tracking a sinusoidal setpoint (a) stator's current $i_{s_{d}}$ (b) stator's current $i_{s_{q}}$ to sensors cost and limited reliability, sensors failures and difficulties in sensors installation) the significance of state estimation through Kalman Filtering is clear. The efficiency of the above mentioned Extended Kalman Filter-based control scheme was tested through simulation experiments.

\section{REFERENCES}

[1] G.G. Rigatos, Adaptive fuzzy control of DC motors using state and output feedback, Electric Power Systems Research, Elsevier, vol. 79, no. 11, pp. 1579-1592, 2009.

[2] J. Holtz, Sensorless control of induction motor drives, Proceedings of the IEEE, vol. 90, no. 8, pp. 1359-1394, 2002.

[3] M. Hilairet, F. Augerb, and E. Berthelot, Speed and rotor flux estimation of induction machines using a two-stage extended Kalman filter, Automatica, Elsevier, vol. 45, pp. 1819-1827, 2009.

[4] B. Akin, U. Orguner, and A. Ersak, Simple derivative-free nonlinear state observer for sensorless AC drives, IEEE/ASME Transactions on Mechatronics, vol. 11, no. 5, pp. 634-643, 2006.

[5] S. Kumar, J. Prakash, and P. Kanagasabapathy, A critical evaluation and experimental verification of Extended Kalman Filter, Unscented Kalman Filter and Neural State Filter for state estimation of three phase induction motor, Applied Soft Computing Journal, Elsevier, vol. 11, no. 3, pp. 31993208, 2011

[6] B. Akin, U. Orguner, and A. Ersak, A comparative study on Kalman filtering techniques designed for state estimation of industrial AC drive systems, Proceedings of the IEEE International Conference on Mechatronics 2004, ICM'04; Istanbul; June 2004.

[7] G.G. Rigatos, Particle and Kalman filtering for state estimation and control of DC motors, ISA Transactions, Elsevier, vol. 48, no. 1, pp. 62-72, 2009.

[8] G.G. Rigatos, Particle Filtering for State Estimation in Nonlinear Industrial Systems, IEEE Transactions on Instrumentation and Measurement, vol. 58, no.11, pp. 3885-3901, 2009.

[9] E.W. Kamen, and J.K. Su, Introduction to Optimal Estimation, Springer, 1999.

[10] M. Bassevile and I. Nikoforov, Detection of abrupt changes: Theory and Applications, Prentice-Hall, 1993.

[11] G.G. Rigatos and S.G. Tzafestas, Extended Kalman Filtering for Fuzzy Modelling and Multi-Sensor Fusion, Mathematical and Computer Modelling of Dynamical Systems, Taylor \& Francis, vol. 13, pp. 251-266, 2007.

[12] G. Rigatos and Q. Zhang, Fuzzy Model Validation using the Local Statistical Approach, Publication Interne IRISA No 1417, Rennes, France, 2001.

[13] R. Marino, S. Peresada and P. Valigi, Adaptive Input-Output Linearizing Control of Induction Motors, IEEE Transactions on Automatic Control, vol. 38, no.2, pp. 208-221, 1991.

[14] P. Martin and P. Rouchon, Two remarks on induction motors, CESA '96 IMACS Multiconference, vol. 1, pp. 76-79, Lille, France, 1996.

[15] E. Delaleau, J.P. Louis and R. Ortega, Modelling and Control of Induction Motors, International Journal of Applied Mathematics and Computer Science, vol.11, no.1, pp.105-129, 2001

[16] J. Villagra, B. d'Andrea-Novel, H. Mounier and M. Pengov, Flatnessbased vehicle steering control strategy with SDRE feedback gains tuned via a sensitivity approach, IEEE Transactions on Control Systems Technology, vol. 15, pp. 554- 565, 2007.

[17] J. Dannehl and F.W. Fuchs, Flatness-based control of an induction machine fed via voltage source inverter - Concept, control design and performance analysis, IECON 2006, IEEE Conference on Industrial Electronics, pp. 5125-5130, 2006.

[18] G.G. Rigatos, Modelling and Control for Intelligent Industrial Systems: Adaptive Algorithms in Robotics and Industrial Engineering, Springer, 2011.

[19] F. Alonge, and A. D'Ippolito, Robustness analysis of an Extended Kalman Filter for sensorless control of induction motors 2010 IEEE International Symposium on Industrial Electronics, ISIE 2010, Bari, Italy, July 2010.

[20] G. Besançon, Q. Zhang, and H. Hammouri, High-gain observer based state and parameter estimation in nonlinear systems, IFAC Symposium on Nonlinear Control Systems (NOLCOS), Stuttgart, Germany, 2004. 